IFIC/05-37

hep-ph/0508138

\title{
Multigluonic scattering amplitudes of heavy quarks
}

\author{
Germán Rodrigo ${ }^{(a) *}$ \\ (a) Instituto de Física Corpuscular, CSIC-Universitat de València, \\ Apartado de Correos 22085, E-46071 Valencia, Spain.
}

\begin{abstract}
We consider heavy quark and antiquark off-shell spinorial currents with emission of an arbitrary number of gluons of positive helicity. From this results we calculate the corresponding on-shell scattering amplitude and the quark-antiquark vector current. Then, we show that in the heavy top quark effective theory the holomorfic component of the Higgs $\rightarrow q \bar{q}+n$-gluon amplitude vanish for helicity conserving configurations.
\end{abstract}

IFIC/05-37

August 11, 2005

*E-mail: german.rodrigo@ific.uv.es 


\section{Introduction}

Production of jets at large transverse momentum with respect to the beam is the typical signature at high energy hadron colliders, both for signal and background. The LHC will produce an enormous amount of heavy quarks. Indeed it can be considered a heavy quark factory. Since heavy quarks will appear associated to jets, the calculation of multipartonic scattering amplitudes with heavy fermions is mandatory in order to provide accurate phenomenological predictions. But the complexity of perturbative calculations increases exponentially with the number of jets, in such a way that the usual expansion in Feynman diagrams becomes prohibitive. Recursion relations within the helicity amplitude formalism [1, 2, 3] have been proven to be an elegant and efficient tool to calculate multipartonic scattering amplitudes, and might allow to overcome this problem. Together with the calculation of the scattering amplitudes, more efficient methods to integrate over the intricate multipartonic phase-space are also required for the development of Monte Carlo event generators.

Recursion relations have been extensively used in the literature at tree [4, 5] and one-loop level [6, 7] in the past. Witten's idea of a duality between supersymmetric Yang-Mills and topological string theories in twistor space [8] has reopened the interest in this issue. Since then, a new method for the evaluation of scattering amplitudes in gauge theories has been proposed [9]. It is based on the recursive use of offshell Maximal Helicity Violating amplitudes (MHV) [10]. Recent works have accomplished interesting progress since the original formulation, and the method has been refined by introducing more efficient recursion relations [11, 12], and extending this approach to the one-loop level [13, 14]. Although initially formulated for massless particles the MHV rules have been generalized to include heavy particles. Within this formalism recursion relations for massive scalar particles at tree-level have been introduced in Ref. [16] and for vector boson and fermions in Ref. [15]. Those recursion relations have been used by the authors of Ref. [17] to calculate multigluonic amplitudes with heavy scalars.

Due to the phenomenological impact of heavy quark production at LHC, we analyze in this paper the potentiality of the Berends-Giele recursion relations [4] to evaluate several helicity amplitudes with heavy quarks and an arbitrary number of gluons. We obtain compact expressions for some helicity configurations. We find that this method provides efficiently valuable information for the calculation of scattering amplitudes with heavy particles, in a complementary way to the MHV approach.

\section{Scattering amplitudes with one quark-antiquark pair}

Spinors for massive fermions can be constructed from two null vectors [18, 19, 20]. The decomposition is however not unique, and the freedom to choose such vectors makes the helicity amplitude method very powerful also for massive particles. We shall consider processes where quark-antiquark pairs are

produced. We denote by $p_{1}^{\mu}$ and $p_{2}^{\mu}$, with $p_{1}^{2}=p_{2}^{2}=m^{2}$, the end point four-momentum of the fermion line. In terms of two light-like vectors $\left(\hat{p}_{1}^{2}=\hat{p}_{2}^{2}=0\right)$ the outgoing fermion-antifermion four-momenta can be written as

$$
\begin{aligned}
& p_{1}^{\mu}=\frac{1+\beta}{2} \hat{p}_{1}^{\mu}+\frac{1-\beta}{2} \hat{p}_{2}^{\mu}, \\
& p_{2}^{\mu}=\frac{1-\beta}{2} \hat{p}_{1}^{\mu}+\frac{1+\beta}{2} \hat{p}_{2}^{\mu},
\end{aligned}
$$


where $\beta=\sqrt{1-4 m^{2} / s_{12}}$ is the velocity of the quark, and $s_{12}=\left(p_{1}+p_{2}\right)^{2}$. This momentum transformation is of course not unique. The advantage of our choice of normalization is that it preserves momentum conservation since trivially $\hat{p}_{1}+\hat{p}_{2}=p_{1}+p_{2}$, and hence $\hat{s}_{12}=\left(\hat{p}_{1}+\hat{p}_{2}\right)^{2}=s_{12}$. Furthermore, in the massless limit we have: $p_{1} \rightarrow \hat{p}_{1}$ and $p_{2} \rightarrow \hat{p}_{2}$. The inverse transformation reads:

$$
\begin{aligned}
& \hat{p}_{1}^{\mu}=\frac{1+\beta}{2 \beta} p_{1}^{\mu}-\frac{1-\beta}{2 \beta} p_{2}^{\mu}, \\
& \hat{p}_{2}^{\mu}=-\frac{1-\beta}{2 \beta} p_{1}^{\mu}+\frac{1+\beta}{2 \beta} p_{2}^{\mu} .
\end{aligned}
$$

Then, we use $\hat{p}_{1}$ and $\hat{p}_{2}$ as reference null vectors to define the quark-antiquark spinors:

$$
\bar{u}_{ \pm}\left(p_{1}, m\right)=\frac{\beta_{+}^{-1 / 2}}{\left\langle 2^{\mp} \mid 1^{ \pm}\right\rangle}\left\langle 2^{\mp}\left|\left(\not p_{1}+m\right), \quad v_{ \pm}\left(p_{2}, m\right)=\frac{\beta_{+}^{-1 / 2}}{\left\langle 2^{\mp} \mid 1^{ \pm}\right\rangle}\left(\not p_{2}-m\right)\right| 1^{ \pm}\right\rangle
$$

where $\beta_{ \pm}=(1 \pm \beta) / 2$, and we use the shorthand notation $\left|i^{ \pm}\right\rangle=\left|\hat{p}_{i}^{ \pm}\right\rangle$. With this definition the massive spinors have a smooth massless limit. For $m=0$ :

$$
\bar{u}_{ \pm}\left(p_{1}\right)=\left\langle 1^{ \pm}\left|, \quad v_{ \pm}\left(p_{2}\right)=\right| 2^{\mp}\right\rangle .
$$

We consider now a quark-antiquark vector current. In the helicity basis, we find:

$$
S^{\mu}\left(1_{q}^{ \pm}, 2_{\bar{q}}^{\mp}\right)=\bar{u}_{ \pm}\left(p_{1}, m\right) \gamma^{\mu} v_{\mp}\left(p_{2}, m\right)=\left\langle 1^{ \pm}\left|\gamma^{\mu}\right| 2^{ \pm}\right\rangle .
$$

Namely, the vector current has the same functional form as the massless one but replacing the massless momenta by the corresponding null vectors used to define the massive spinors. For massive fermions, we shall consider also the case of helicity flip along the fermion line, which would vanish in the massless limit. We obtain:

$$
S^{\mu}\left(1_{q}^{ \pm}, 2_{\bar{q}}^{ \pm}\right)=\bar{u}_{ \pm}\left(p_{1}, m\right) \gamma^{\mu} v_{ \pm}\left(p_{2}, m\right)=\frac{2 m}{\left\langle 2^{\mp} \mid 1^{ \pm}\right\rangle}\left(\hat{p}_{2}-\hat{p}_{1}\right)^{\mu}
$$

Generalizing these results to the insertion of an arbitrary odd number of gamma matrices between the spinors, the following useful properties hold:

$$
\begin{aligned}
S^{\mu_{1} \ldots \mu_{2 n+1}}\left(1_{q}^{ \pm}, 2_{\bar{q}}^{\mp}\right) & =\bar{u}_{ \pm}\left(p_{1}, m\right) \gamma^{\mu_{1}} \cdots \gamma^{\mu_{2 n+1}} v_{\mp}\left(p_{2}, m\right) \\
& =\beta_{+}\left\langle 1^{ \pm}\left|\gamma^{\mu_{1}} \cdots \gamma^{\mu_{2 n+1}}\right| 2^{ \pm}\right\rangle+\beta_{-}\left\langle 1^{ \pm}\left|\gamma^{\mu_{2 n+1}} \cdots \gamma^{\mu_{1}}\right| 2^{ \pm}\right\rangle,
\end{aligned}
$$

and

$$
\begin{aligned}
S^{\mu_{1} \ldots \mu_{2 n+1}}\left(1_{q}^{ \pm}, 2_{\bar{q}}^{ \pm}\right) & =\bar{u}_{ \pm}\left(p_{1}, m\right) \gamma^{\mu_{1}} \cdots \gamma^{\mu_{2 n+1}} v_{ \pm}\left(p_{2}, m\right) \\
& =\frac{m}{\left\langle 2^{\mp} \mid 1^{ \pm}\right\rangle}\left(\left\langle 2^{\mp}\left|\gamma^{\mu_{1}} \cdots \gamma^{\mu_{2 n+1}}\right| 2^{\mp}\right\rangle-\left\langle 1^{ \pm}\left|\gamma^{\mu_{1}} \cdots \gamma^{\mu_{2 n+1}}\right| 1^{ \pm}\right\rangle\right) .
\end{aligned}
$$

In the massless limit $\beta_{+} \rightarrow 1$ and $\beta_{-} \rightarrow 0$, and only the first term in Eq. (7) survives, while Eq. (8) obviously vanishes. Similar relations can be derived for the insertion of an even number of gamma matrices as well, but those are not needed for the current purpose of this paper.

In the following we will consider the quark-antiquark vector current, with emission of an arbitrary number of gluons $V^{\mu} \rightarrow q \bar{q}+(n-2) g$. The colour decomposition is such that

$$
\hat{S}^{\mu}\left(1_{q} ; 2,3, \ldots, n-1 ; n_{\bar{q}}\right)=g_{\mathrm{S}}^{n-2} \sum_{P(2, \ldots, n-1)}\left(T^{a_{2}} T^{a_{3}} \cdots T^{a_{n-1}}\right) S^{\mu}\left(1_{q} ; 2,3, \ldots, n-1 ; n_{\bar{q}}\right),
$$

where $P(2, \ldots, n-1)$ is the permutation group of all the gluons, and $T^{a_{i}}$ are colour matrices in the fundamental representation. 


\section{Spinorial off-shell currents}

Recursion relations for the calculation of the spinorial current of a quark-antiquark pair and $n$-gluons, where either the quark or the antiquark are off-shell, have been derived long time ago [4]. Those recursion relations are valid regardless the massive or massless character of the quark-antiquark pair, although explicit results where obtained only in the massless approximation. In this Section, we use these recursion relations to extend their results to the heavy quark case.

The colour ordered spinorial current of an on-shell quark of four-momentum $p_{1}$ and $(m-1)$-gluons of four-momenta $p_{2}$ to $p_{m}$ is given in terms of the spinorial current of the on-shell quark with less gluons, and the off-shell gluonic current $J^{\mu}$ of the rest of the gluons:

$$
S\left(1_{q} ; 2, \ldots, m\right)=-\sum_{k=1}^{m-1} S\left(1_{q} ; 2, \ldots, k\right) \mathcal{J}(k+1, \ldots, m) \frac{1}{\not p_{1, m}-m},
$$

where $p_{1, m}=p_{1}+p_{2}+\ldots+p_{m}$ and $S\left(1_{q}\right)=\bar{u}\left(p_{1}, m\right)$. For all gluons of positive helicity the gluonic current has the form [4]:

$$
J^{\mu}\left(i^{+}, \ldots, j^{+}\right)=\frac{\left\langle\xi\left|\gamma^{\mu} \not p_{i, j}\right| \xi\right\rangle}{\sqrt{2}\langle\xi i\rangle\langle i(i+1)\rangle \cdots\langle j \xi\rangle} .
$$

The null vector $\xi$ is the reference gauge vector which is assumed to be the same for all the gluons. Conversely, for the off-shell quark current one has

$$
S\left(m+1, \ldots, n-1 ; n_{\bar{q}}\right)=\frac{1}{\not p_{m+1, n}+m} \sum_{k=m+2}^{n} \mathcal{J}(m+1, \ldots, k-1) S\left(k, \ldots, n-1 ; n_{\bar{q}}\right),
$$

the antiquark carrying four-momentum $p_{n}$, and $S\left(n_{\bar{q}}\right)=v\left(p_{n}, m\right)$.

It is convenient to work in the gauge where $\xi=\hat{p}_{n}$, where $\hat{p}_{n}$ is the reference null vector of the antiquark. In that gauge, diagrams with gluons of positive helicity attached to an antiquark of negative helicity do not contribute in the massless approximation. This is not the case for massive quarks, but that choice of gauge still provides some simplifications. Furthermore: $m^{2}=\beta_{+} \beta_{-}\langle 1 \xi\rangle[\xi 1]$. For the complementary helicity configurations it would be convenient to choose $\xi=\hat{p}_{1}$ instead.

The no-gluon, one-gluon, and two-gluon spinorial quark currents are

$$
\begin{gathered}
S\left(1_{q}^{+}\right)=\bar{u}_{+}\left(p_{1}, m\right)=\frac{\beta_{+}^{-1 / 2}}{\langle\xi 1\rangle}\langle\xi|\left(\not p_{1}+m\right)=\beta_{+}^{1 / 2}\left[1 \mid+\beta_{+}^{-1 / 2} \frac{m}{\langle\xi 1\rangle}\langle\xi|,\right. \\
S\left(1_{q}^{+} ; 2^{+}\right)=-S\left(1_{q}^{+}\right) \oiint_{2}^{+} \frac{1}{\not p_{12}-m}=-S\left(1_{q}^{+}\right) \frac{\mid 2]}{y_{12}\langle\xi 2\rangle}\langle\xi|\left(\not p_{12}+m\right), \\
S\left(1_{q}^{+} ; 2^{+}, 3^{+}\right)=-\left(S\left(1_{q}^{+} ; 2^{+}\right) \oiint_{3}^{+}+S\left(1_{q}^{+}\right) \mathcal{J}\left(2^{+}, 3^{+}\right)\right) \frac{1}{\not \phi_{1,3}-m} \\
=\frac{S\left(1_{q}^{+}\right)}{y_{1,3}\langle\xi 2\rangle\langle\xi 3\rangle}\left(\frac{\mid 2]}{y_{12}}\left\langle\xi\left|\not p_{12}\right| 3\right]+\frac{\not \phi_{23}|\xi\rangle}{\langle 23\rangle}\right)\langle\xi|\left(\not p_{1,3}+m\right),
\end{gathered}
$$


where we defined $y_{1, n}=p_{1, n}^{2}-m^{2}$. After some algebra we can simplify further the two-gluon current

$$
S\left(1_{q}^{+} ; 2^{+}, 3^{+}\right)=-\frac{S\left(1_{q}^{+}\right)}{\langle 23\rangle y_{12}}\left(\frac{\mid 2]}{\langle\xi 3\rangle}+\beta_{-} \frac{\mid \xi][23]}{y_{1,3}}\right)\langle\xi|\left(\not p_{1,3}+m\right) .
$$

Then, we factorize the off-shell current into the form

$$
S\left(1_{q}^{+} ; 2^{+}, \ldots, n^{+}\right)=-\frac{S\left(1_{q}^{+}\right)}{y_{12}} X\left(1_{q}^{+} ; 2^{+}, \ldots, n^{+}\right)\langle\xi|\left(\not \phi_{1, n}+m\right),
$$

where

$$
\begin{aligned}
X\left(1_{q}^{+} ; 2^{+}\right) & =\frac{\mid 2]}{\langle\xi 2\rangle}, \\
X\left(1_{q}^{+} ; 2^{+}, 3^{+}\right) & =\frac{1}{\langle 23\rangle}\left(\frac{\mid 2]}{\langle\xi 3\rangle}+\beta_{-} \frac{\mid \xi][23]}{y_{1,3}}\right) .
\end{aligned}
$$

The massless limit forces the off-shell massive current to have the following structure

$$
\left.X\left(1_{q}^{+} ; 2^{+}, \ldots, n^{+}\right)=\frac{1}{\langle 23\rangle\langle 34\rangle \ldots\langle(n-1) n\rangle}\left[\frac{\mid 2]}{\langle\xi n\rangle}+\beta_{-} \mid \xi\right] A\left(1_{q}^{+} ; 2^{+}, \ldots, n^{+}\right)\right]
$$

From Eq. (12), we can easily derive a recursion relation for the $X$ factor of the off-shell current

$$
\begin{aligned}
X\left(1_{q}^{+} ; 2^{+}, \ldots, n^{+}\right) & =\frac{1}{\langle\xi n\rangle y_{1, n}}\left[-\frac{\not \not_{2, n}|\xi\rangle y_{12}}{\langle\xi 2\rangle\langle 23\rangle \ldots\langle(n-1) n\rangle}\right. \\
& +\sum_{k=2}^{n-2} X\left(1_{q}^{+} ; 2^{+}, \ldots, k^{+}\right) \frac{\left\langle\xi\left|\not p_{1, k} \not p_{k+1, n}\right| \xi\right\rangle}{\langle\xi(k+1)\rangle\langle(k+1)(k+2)\rangle \ldots\langle(n-1) n\rangle} \\
& \left.-X\left(1_{q}^{+} ; 2^{+}, \ldots, n-1^{+}\right)\left\langle\xi\left|\not p_{1, n-1}\right| n\right]\right] .
\end{aligned}
$$

To simplify this recursion relation further and derive an equivalent recursion relation for the function $A$, we multiply and divide the last term of Eq. (20) by $\langle(n-1) n\rangle$, such that

$$
\begin{aligned}
\left\langle\xi\left|\not p_{1, n-1}\right| n\right]\langle(n-1) n\rangle & \left.=\left\langle n-1\left|\not p_{n} \not \phi_{1, n-1}\right| \xi\right\rangle=\langle n-1|\left(\not p_{1, n}-\not \phi_{1, n-2}\right) \not p_{1, n}\right)|\xi\rangle \\
& =\left\langle n-1\left|p_{1, n}^{2}-\not \phi_{1, n-2}\left(\not p_{1, n-2}+\not p_{n-1, n}\right)\right| \xi\right\rangle \\
& =-y_{1, n}\langle\xi(n-1)\rangle-\left\langle n-1\left|y_{1, n-2}+\not p_{1, n-2} \not p_{n-1, n}\right| \xi\right\rangle .
\end{aligned}
$$

The first term in the rhs of Eq. (21) generates the first term of the rhs of Eq. (19). Then, it is clear that in order to fulfill the massless limit, the remaining contributions have to either cancel to each other or be proportional to $\beta_{-}$. The proof follows by induction. Assuming that Eq. (19) is true for $n-1$, we show that it is also true for $n$ through the recursion relation in Eq. (20). For that, we use recursively the Fierz identity:

$$
\langle k|=\frac{1}{\langle\xi(k-1)\rangle}(\langle k(k-1)\rangle\langle\xi|+\langle\xi k\rangle\langle k-1|),
$$

and

$$
\left\langle k\left|y_{1, k}+\not \not_{1, k} \not p_{k+1, n}\right| \xi\right\rangle=\left\langle k\left|y_{1, k-1}+\not \not_{1, k-1} \not p_{k, n}\right| \xi\right\rangle
$$


This results into the following recursion relation for the corresponding $A$ function:

$$
A\left(1_{q}^{+} ; 2^{+}, \ldots, n^{+}\right)=\frac{1}{\langle\xi n\rangle y_{1, n}}\left[\sum_{k=2}^{n-1} A\left(1_{q}^{+} ; 2^{+}, \ldots, k^{+}\right) \frac{\langle k(k+1)\rangle}{\langle\xi(k+1)\rangle}\left\langle\xi\left|\not p_{1, k} \not \phi_{k+1, n}\right| \xi\right\rangle-\left[2\left|\not \phi_{3, n}\right| \xi\right\rangle\right],
$$

where

$$
A\left(1_{q}^{+} ; 2^{+}\right)=0, \quad A\left(1_{q}^{+} ; 2^{+}, 3^{+}\right)=\frac{[23]}{y_{1,3}}
$$

From Eq. (24) we find

$$
A\left(1_{q}^{+} ; 2^{+}, 3^{+}, 4^{+}\right)=A\left(1_{q}^{+} ; 2^{+}, 3^{+}\right)\left(\frac{\langle\xi 3\rangle}{\langle\xi 4\rangle}+\frac{\left[2\left|\not \not_{1} \not p_{23}\right| 4\right]}{[23] y_{1,4}}\right) .
$$

To obtain this result we have transformed he last term in the rhs of Eq. (24) in the following way

$$
\left[2\left|\not p_{3, n}\right| \xi\right\rangle=A\left(1_{q}^{+} ; 2^{+}, 3^{+}\right) \frac{\left[2\left|y_{1,3} \not \not_{3, n}\right| \xi\right\rangle}{[23]}=A\left(1_{q}^{+} ; 2^{+}, 3^{+}\right)\left(\frac{\left[2\left|\not p_{1} \not p_{23} \not \not p_{4, n}\right| \xi\right\rangle}{[23]}+\left\langle 3\left|\not p_{12} \not p_{3, n}\right| \xi\right\rangle\right)
$$

Assuming that for an arbitrary number of gluons larger than two the $A$ function has the form

$$
A\left(1_{q}^{+} ; 2^{+}, \ldots, n^{+}\right)=A\left(1_{q}^{+} ; 2^{+}, 3^{+}\right)\left(\frac{\langle\xi 3\rangle}{\langle\xi n\rangle}+\frac{\bar{A}\left(1_{q}^{+} ; 2^{+}, \ldots, n^{+}\right)}{[23]}\right)
$$

the $\bar{A}$ function fulfills the recursion relation

$$
\bar{A}\left(1_{q}^{+} ; 2^{+}, \ldots, n^{+}\right)=\frac{1}{\langle\xi n\rangle y_{1, n}}\left[\sum_{k=4}^{n-1} \bar{A}\left(1_{q}^{+} ; 2^{+}, \ldots, k^{+}\right) \frac{\langle k(k+1)\rangle}{\langle\xi(k+1)\rangle}\left\langle\xi\left|\not p_{1, k} \not p_{k+1, n}\right| \xi\right\rangle-\left[2\left|\not p_{1} \not p_{23} \not \not_{4, n}\right| \xi\right\rangle\right] .
$$

By solving this equation recursively, we get finally

$$
\begin{aligned}
\bar{A}\left(1_{q}^{+} ; 2^{+}, \ldots, n^{+}\right)=\sum_{j=1}^{n-4} \frac{\bar{A}\left(1_{q}^{+} ; 2^{+}, 3^{+}, 4^{+}\right)\langle 45\rangle\left\langle\xi\left|\not p_{1,4} \not p_{5, w_{1}}\right| \xi\right\rangle-\langle\xi 5\rangle\left[2\left|\not p_{1} \not p_{23} \not p_{4, w_{1}}\right| \xi\right\rangle}{y_{1, w_{1}} \cdots y_{1, w_{j}}} \\
\times \frac{\left\langle w_{1}\left(w_{1}+1\right)\right\rangle \cdots\left\langle w_{j-1}\left(w_{j-1}+1\right)\right\rangle\left\langle\xi\left|\not p_{1, w_{1}} \not p_{w_{1}+1, w_{2}}\right| \xi\right\rangle \cdots\left\langle\xi\left|\not p_{1, w_{j-1}} \not p_{w_{j-1}+1, w_{j}}\right| \xi\right\rangle}{\langle\xi 5\rangle\left\langle\xi w_{1}\right\rangle\left\langle\xi\left(w_{1}+1\right)\right\rangle \cdots\left\langle\xi w_{j-1}\right\rangle\left\langle\xi\left(w_{j-1}+1\right)\right\rangle\langle\xi n\rangle}
\end{aligned}
$$

where $w_{k} \epsilon\{5, n\}$, and $w_{1}<w_{2}<\ldots<w_{j}$ with $w_{j}=n$. This completes the calculation of the off-shell quark spinorial current.

\subsection{Antiquark off-shell current}

Very similar results can be obtained for the antiquark spinorial current when the antiquark carries negative helicity. The no-gluon, one-gluon and two-gluon currents are given by

$$
\left.S\left(n_{\bar{q}}^{-}\right)=\beta_{+}^{1 / 2}|\xi\rangle+\beta_{+}^{-1 / 2} \frac{m}{[1 \xi]} \mid 1\right]=-\frac{\beta_{+}^{1 / 2}}{m}\left(\not p_{n}-m\right)|\xi\rangle
$$




$$
\begin{gathered}
S\left(2^{+} ; 3_{\bar{q}}^{-}\right)=\left(\not \not_{23}-m\right)|\xi\rangle \frac{[2 \mid}{\langle\xi 2\rangle y_{23}} S\left(3_{\bar{q}}^{-}\right) \\
S\left(2^{+}, 3^{+} ; 4_{\bar{q}}^{-}\right)=\left(\not p_{2,4}-m\right)|\xi\rangle\left(\frac{[3 \mid}{\langle\xi 2\rangle}-\beta_{+} \frac{[23][\xi \mid}{y_{2,4}}\right) \frac{S\left(4_{\bar{q}}^{-}\right)}{\langle 23\rangle y_{34}} .
\end{gathered}
$$

For an arbitrary number of gluons, we have in general

$$
S\left(k^{+}, \ldots, n-1^{+} ; n_{\bar{q}}^{-}\right)=(\not p k, n-m)|\xi\rangle X\left(k^{+}, \ldots, n-1^{+} ; n_{\bar{q}}^{-}\right) \frac{S\left(n_{\bar{q}}^{-}\right)}{y_{n-1, n}},
$$

with

$$
\begin{aligned}
X\left(k^{+}, \ldots, n-1^{+} ; n_{\bar{q}}^{-}\right) & =\frac{1}{\langle k(k+1)\rangle \cdots\langle(n-2)(n-1)\rangle} \\
& \times\left[\frac{[n-1 \mid}{\langle\xi k\rangle}-\beta_{+}\left[\xi \mid A\left(k^{+}, \ldots, n-1^{+} ; n_{\bar{q}}^{-}\right)\right]\right.
\end{aligned}
$$

where

$$
A\left(k^{+}, \ldots, n-1^{+} ; n_{\bar{q}}^{-}\right)=A\left(n-2^{+}, n-1^{+} ; n_{\bar{q}}^{-}\right)\left(\frac{\langle\xi(n-2)\rangle}{\langle\xi k\rangle}+\bar{A}\left(k^{+}, \ldots, n-1^{+} ; n_{\bar{q}}^{-}\right)\right) .
$$

A recursion relation similar to Eq. (29) can also be obtained for this antiquark current, leading to an expression similar to Eq. (30) where now the labeling of the four-momenta runs backwards.

\section{On-shell scattering amplitudes}

From the spinorial off-shell current one can calculate the amplitude for the process of $n-2$ outgoing gluons and a quark-antiquark pair. This amplitude is obtained from the quark-gluon spinorial current with $n-2$ gluons, by removing the propagator of the off-shell quark, contracting the current with the antiquark spinor and imposing momentum conservation

$$
S\left(1_{q}^{+} ; 2^{+}, \ldots, n-1^{+} ; n_{\bar{q}}^{\mp}\right)=-\frac{y_{1, n-1}}{y_{12}} S\left(1_{q}^{+}\right) X\left(1_{q}^{+} ; 2^{+}, \ldots, n-1^{+}\right)\left\langle\xi\left|S\left(n_{\bar{q}}^{\mp}\right)\right|_{p_{1, n}=0} .\right.
$$

The helicity conserving amplitude annihilates, and for the helicity flip amplitude we get

$$
S\left(1_{q}^{+} ; 2^{+}, \ldots, n-1^{+} ; n_{\bar{q}}^{+}\right)=\left.\frac{m^{3}}{\beta_{+} y_{12}} \frac{y_{1, n-1} A\left(1_{q}^{+} ; 2^{+}, \ldots, n-1^{+}\right)}{\langle 23\rangle\langle 34\rangle \cdots\langle(n-2)(n-1)\rangle\langle n 1\rangle}\right|_{y_{1, n-1}=0}
$$

In particular

$$
S\left(1_{q}^{+} ; 2^{+}, 3^{+} ; 4_{\bar{q}}^{+}\right)=\frac{m^{3}}{\beta_{+} y_{12}} \frac{[23]}{\langle 23\rangle\langle 41\rangle} .
$$




\section{Quark-antiquark vector current}

From the results obtained in the previous sections we can also calculate the off-shell quark-antiquark vector current

$$
S^{\mu}\left(1_{q} ; 2, \ldots, n-1 ; n_{\bar{q}}\right)=\sum_{k=1}^{n-1} S\left(1_{q} ; 2, \ldots, k\right) \gamma^{\mu} S\left(k+1, \ldots, n-1 ; n_{\bar{q}}\right) .
$$

For all gluons of positive helicity all the terms contributing to the sum in Eq. (40) have the form

$$
S_{k}^{\mu}\left(1_{q}^{+} ; 2^{+}, \ldots, n-1^{+} ; n_{\bar{q}}^{\mp}\right) \propto\left\langle\xi\left|\left(\not \phi_{1, k}+m\right) \gamma^{\mu}\left(\not \phi_{k+1, n}-m\right)\right| \xi^{ \pm}\right\rangle .
$$

Then, after some algebra we find that

$$
\begin{aligned}
& S_{k}^{\mu}\left(1_{q}^{+} ; 2^{+}, \ldots, n-1^{+} ; n_{\bar{q}}^{-}\right) \propto-m\left\langle\xi\left|\not p_{1, n-1} \gamma^{\mu}\right| \xi\right\rangle, \\
& S_{k}^{\mu}\left(1_{q}^{+} ; 2^{+}, \ldots, n-1^{+} ; n_{\bar{q}}^{+}\right) \propto\left\langle\xi\left|\not p_{1, k} \gamma^{\mu} \not p_{k+1, n}\right| \xi\right]-2 m^{2} \xi^{\mu},
\end{aligned}
$$

Particularly interesting is the helicity conserving current. In that case all the terms are proportional to the same quantity, where now the quark four-momentum has been replaced by its reference null vector $\hat{p}_{1}$. Since in Eq. (42) only massless vectors appear some properties that hold for massless scattering amplitudes can be generalized to the massive case directly without further proof. For some applications see Section 6 . We obtain the following expression for the helicity conserving vector current:

$$
\begin{aligned}
S^{\mu}\left(1_{q}^{+} ;\right. & \left.2^{+}, \ldots, n-1^{+} ; n_{\bar{q}}^{-}\right)=\frac{1}{\langle 23\rangle\langle 34\rangle \cdots\langle(n-2)(n-1)\rangle}\left\{\frac{\beta_{+}[12]}{\langle\xi(n-1)\rangle y_{12}}-\frac{\beta_{-}[1(n-1)]}{\langle\xi 2\rangle y_{n-1, n}}\right. \\
& +\frac{m^{2}}{\langle\xi 1\rangle}\left[\frac{1}{y_{12}} A\left(1_{q}^{+} ; 2^{+}, \ldots, n-1^{+}\right)+\frac{1}{y_{n-1, n}} A\left(2^{+}, \ldots, n-1^{+} ; n_{\bar{q}}^{-}\right)\right] \\
& -\frac{1}{y_{12} y_{n-1, n}} \frac{m^{2}}{11 \xi]} \sum_{k=2}^{n-2}\langle k(k+1)\rangle\left[\frac{[12]}{\langle\xi k\rangle}+\beta_{-}[1 \xi] A\left(1_{q}^{+} ; 2^{+}, \ldots, k^{+}\right)\right] \\
& \left.\times\left[\frac{[(n-1) 1]}{\langle\xi(k+1)\rangle}+\beta_{+}[1 \xi] A\left(k+1^{+}, \ldots, n-1^{+} ; n_{\bar{q}}^{-}\right)\right]\right\}\left\langle\xi\left|\not_{1, n-1} \gamma^{\mu}\right| \xi\right\rangle .
\end{aligned}
$$

In the massless limit, the current in Eq. (44) agrees with the Berends-Giele current [4]:

$$
\left.S^{\mu}\left(1_{q}^{+} ; 2^{+}, \ldots, n-1^{+} ; n_{\bar{q}}^{-}\right)\right|_{m=0}=\frac{\left\langle\xi\left|\not p_{1, n-1} \gamma^{\mu}\right| \xi\right\rangle}{\langle 12\rangle\langle 23\rangle \cdots\langle(n-1) \xi\rangle} .
$$

In the following we present some simple examples.

\subsection{Single gluon emission}

Let us consider a quark-antiquark vector current with emission of one single gluon off the quarkantiquark pair. For the helicity conserving vector current, we find:

$$
S^{\mu}\left(1_{q}^{+} ; 2^{+} ; 3_{\bar{q}}^{-}\right)=\left(\frac{\beta_{+}}{y_{12}}-\frac{\beta_{-}}{y_{23}}\right) \frac{[12]}{\langle\xi 2\rangle}\left\langle\xi\left|\ddot{p}_{12} \gamma^{\mu}\right| \xi\right\rangle=\beta \frac{[1|2| \xi\rangle}{y_{12} y_{23}}\left\langle\xi\left|\not p_{12} \gamma^{\mu}\right| \xi\right\rangle .
$$

The helicity flip configuration is also quite simple, we get

$$
S^{\mu}\left(1_{q}^{+} ; 2^{+} ; 3_{\bar{q}}^{+}\right)=m\left[\left(\frac{\beta_{+}}{y_{12}}-\frac{\beta_{-}}{y_{23}}\right) \frac{[12]}{\langle\xi 2\rangle} 2\left(\hat{p}_{3}-\hat{p}_{1}\right)^{\mu}+\frac{1}{\langle\xi 1\rangle}\left[2\left|\left(\frac{\hat{p}_{1}}{y_{12}}+\frac{\not p_{3}}{y_{23}}\right) \gamma^{\mu}\right| 2\right\rangle\right],
$$

which clearly annihilates in the massless limit. 


\subsection{Two gluons vector current}

The helicity conserving vector current with emission of two gluons is

$$
\begin{aligned}
S^{\mu}\left(1_{q}^{+} ; 2^{+}, 3^{+} ; 4_{\bar{q}}^{-}\right) & =\left[\frac{1}{\langle 23\rangle}\left(\frac{[12] \beta_{+}}{\langle\xi 3\rangle y_{12}}-\frac{\beta_{-}[13]}{y_{34}\langle\xi 2\rangle}\right)-\frac{m^{2}}{y_{12} y_{34}} \frac{[21][31]}{\langle\xi 2\rangle\langle\xi 3\rangle[1 \xi]}\right. \\
& \left.-\frac{m^{2}[32]}{\langle 23\rangle\langle\xi 1\rangle}\left(\frac{1}{y_{12} y_{1,3}}+\frac{1}{y_{34} y_{2,4}}\right)\right]\left\langle\xi\left|\ddot{p}_{123} \gamma^{\mu}\right| \xi\right\rangle .
\end{aligned}
$$

\section{Higgs production together with heavy quarks}

One of the main objectives of the LHC program is the discovery of the Higgs boson. Gluons couple to Higgs through a top quark loop dominantly, where the top can be integrating out leading to an effective interaction. MHV rules for Higgs plus multiparton amplitudes have been presented in Refs. [21, 22] in that effective theory. The naive off-shell continuation of the Higgs vertices depends of the gauge spinor however, and therefore do not work as MHV amplitudes. This problem is solved by splitting the Lagrangean into a self-dual and an anti-self-dual part, and introducing a complex scalar where the Higgs is one of its components $\phi=\frac{1}{2}(H+i A)$.

In the heavy top quark effective theory amplitudes of $\phi$-scalars coupled to a quark-antiquark pair and any number of gluons of positive helicity vanish [22]

$$
A_{n}\left(\phi, q_{1}^{ \pm}, g_{2}^{+}, \ldots, g_{n-1}^{+}, \bar{q}_{n}^{\mp}\right)=0 .
$$

In this section we shall extend this result to the heavy quark case. The proof follows almost straitforward from the amplitudes that we have calculated in Section 5. The helicity conserving vector current of a heavy quark-antiquark pair and $m-2$ gluons is

$$
S^{\mu}\left(1_{q}^{+}, 2^{+}, \ldots, m-1^{+}, n_{\bar{q}}^{-}\right)=N_{2, m-1}\left\langle\xi\left|\not p_{1, m-1} \gamma^{\mu}\right| \xi\right\rangle,
$$

and the vector current of all gluons of positive helicity [4] reads

$$
J^{\mu}\left(m^{+}, \ldots, n-1^{+}\right)=N_{m, n-1}\left\langle\xi\left|\gamma^{\mu} \not p_{m, n-1}\right| \xi\right\rangle .
$$

The normalization factors $N$ are irrelevant for the present proof. What is important is that Eq. (50) has the same functional form in the massive and in the massless case, where $\hat{p}_{1}$ is the null vector used to define the quark spinor. The 3-point $\phi g g$ vertex of two off-shell gluons of momenta $k_{1}$ and $k_{2}$ and Lorentz indices $\mu_{1}$ and $\mu_{2}$ is given by [22]:

$$
V_{\mu_{1} \mu_{2}}^{\phi}\left(k_{1}, k_{2}\right)=g_{\mu_{1} \mu_{2}} k_{1} \cdot k_{2}-k_{1 \mu_{2}} k_{2 \mu_{1}}+i \varepsilon_{\mu_{1} \mu_{2} \nu_{1} \nu_{2}} k_{1}^{\nu_{1}} k_{2}^{\nu_{2}} .
$$

Then, one can compute $A_{n}\left(\phi, q_{1}^{+}, g_{2}^{+}, \ldots, g_{n-1}^{+}, \bar{q}_{n}^{-}\right)$by contracting the vertex with the currents in Eq. (50) and Eq. (51), where $k_{1}=p_{1, m-1}+p_{n}=\hat{p}_{1, m-1}+\hat{p}_{n}$, and $k_{2}=p_{m, n-1}$.

The 3-point $\phi g g$ vertex contracted with the quark-antiquark and the Berends-Giele currents gives

$$
\begin{aligned}
V_{\mu \nu}^{\phi} S^{\mu} J^{\nu} & =\left(k_{1} \cdot k_{2}\right)(S \cdot J)-\left(k_{1} \cdot J\right)\left(k_{2} \cdot S\right) \\
& +\frac{1}{4} \sum_{i=m}^{n-1}\left(\left\langle i\left|\gamma^{\mu} \gamma^{\nu} \not k_{1}\right| i\right]+\left[i\left|\gamma^{\mu} \gamma^{\nu} \not k_{1}\right| i\right\rangle\right) S_{\mu} J_{\nu} .
\end{aligned}
$$


Fierzing the gluonic current

$$
\boldsymbol{y}=2 N_{m, n-1} \sum_{j=m}^{n-1}(\mid j]\langle\xi|+| \xi\rangle[j \mid)\langle j \xi\rangle,
$$

and after some algebra, we find

$$
V_{\mu \nu}^{\phi} S^{\mu} J^{\nu}=N_{m, n-1} \frac{k_{2}^{2}}{2}\left\langle\xi\left|\left[S, \not \phi_{1}\right]\right| \xi\right\rangle
$$

where $\left[S, \not \not_{1}\right]$ is the commutator of the quark-antiquark current with its total momentum. Fierzing the quark-antiquark current

$$
S=2 N_{2, m-1} \sum_{i=1}^{m-1}(\mid i]\langle\xi|+| \xi\rangle[i \mid)\langle\xi i\rangle,
$$

the 3-point vertex obviously cancels since in Eq. [55]: $\langle\xi| S=0$ and $S|\xi\rangle=0$.

\section{Conclusions}

We have calculated several tree-level multigluonic helicity amplitudes with heavy quarks. Our approach is based on the Berends-Giele recursive relations. We have shown that the method can be easily applied for the evaluation of multipartonic scattering amplitudes with heavy particles, and leads to compact results. We have considered scattering amplitudes with all gluons of the same helicity only, but other helicity configurations can be calculated efficiently as well. Furthermore, interesting phenomenological implications can be derived from the general structure of multipartonic amplitudes.

\section{Acknowledgements}

This work has been supported by Ministerio de Educación y Ciencia (MEC) under grant FPA200400996 (PARSIFAL), Acciones Integradas DAAD-MEC (contract HA03-164), and Generalitat Valenciana (GV05-015, GV04B-594 and GRUPOS03/013).

\section{A Spinors and polarization vectors}

Massive spinors of four-momentum $p^{\mu}$ and mass $m$ can be constructed with the help of two null vectors $\hat{p}^{\mu}$ and $\hat{q}^{\mu}$ as follows:

$$
\begin{array}{ll}
u_{ \pm}(p, m)=\frac{N}{\left\langle\hat{p}^{\mp} \mid \hat{q}^{ \pm}\right\rangle}(\not p+m)\left|\hat{q}^{ \pm}\right\rangle, & \bar{u}_{ \pm}(p, m)=\frac{N}{\left\langle\hat{q}^{\mp} \mid \hat{p}^{ \pm}\right\rangle}\left\langle\hat{q}^{\mp}\right|(\not p+m), \\
v_{ \pm}(p, m)=\frac{N}{\left\langle\hat{p}^{\mp} \mid \hat{q}^{ \pm}\right\rangle}(\not p-m)\left|\hat{q}^{ \pm}\right\rangle, & \bar{v}_{ \pm}(p, m)=\frac{N}{\left\langle\hat{q}^{\mp} \mid \hat{p}^{ \pm}\right\rangle}\left\langle q^{\mp}\right|(\not p-m),
\end{array}
$$


where $N=(p \cdot \hat{q} / \hat{p} \cdot \hat{q})^{-1 / 2}$ is the normalization factor. Those spinors trivially fulfill the Dirac equation:

$$
(\not p-m) u(p, m)=0, \quad(\not p+m) v(p, m)=0,
$$

and the completeness relation

$$
\sum_{\lambda= \pm} u_{-\lambda} \bar{u}_{\lambda}=\not p+m, \quad \sum_{\lambda= \pm} v_{-\lambda} \bar{v}_{\lambda}=\not p-m
$$

The polarization vector of an outgoing gluon of momentum $k^{\mu}$ is defined as

$$
\varepsilon_{\mu}^{ \pm}(k, \xi)= \pm \frac{\left\langle\xi^{\mp}\left|\gamma_{\mu}\right| k^{\mp}\right\rangle}{\sqrt{2}\left\langle\xi^{\mp} \mid k^{ \pm}\right\rangle},
$$

where $\xi^{\mu}$ is an arbitrary gauge reference momentum satisfying $\xi^{2}=0$. The polarization vector contracted with the gamma matrices reads as follows:

$$
\oiint^{ \pm}(k, \xi)= \pm \frac{\sqrt{2}}{\left\langle\xi^{\mp} \mid k^{ \pm}\right\rangle}\left(\left|k^{\mp}\right\rangle\left\langle\xi^{\mp}|+| \xi^{ \pm}\right\rangle\left\langle k^{ \pm}\right|\right) .
$$

Other practical properties helping to simplify calculations are

$$
\left\langle i^{\mp}\left|\oiint^{ \pm}(k, \xi) \not k=0, \quad \not k \ddagger^{ \pm}(k, \xi)\right| i^{ \pm}\right\rangle=0,
$$

and

$$
\left\langle\xi^{\mp}\left|\phi_{ \pm}(k, \xi)=0, \quad \phi_{ \pm}(k, \xi)\right| \xi^{ \pm}\right\rangle=0 .
$$

Finally, if we choose the reference momenta for all gluons to be the same, then it follows that

$$
\varepsilon^{ \pm}\left(k_{i}, \xi\right) \cdot \varepsilon^{ \pm}\left(k_{j} \cdot \xi\right)=0
$$

\section{References}

[1] M. Jacob and G. C. Wick, Annals Phys. 7 (1959) 404 [Annals Phys. 281 (2000) 774].

[2] J. D. Bjorken and M. C. Chen, Phys. Rev. 154 (1966) 1335.

[3] M. L. Mangano and S. J. Parke, Phys. Rept. 200 (1991) 301.

[4] F. A. Berends and W. T. Giele, Nucl. Phys. B 306 (1988) 759.

[5] L. J. Dixon, arXiv:hep-ph/9601359.

[6] Z. Bern, L. J. Dixon, D. C. Dunbar and D. A. Kosower, Nucl. Phys. B 425, 217 (1994) [arXiv:hep-ph/9403226].

[7] Z. Bern, L. J. Dixon, D. C. Dunbar and D. A. Kosower, Nucl. Phys. B 435, 59 (1995) [arXiv:hep-ph/9409265].

[8] E. Witten, Commun. Math. Phys. 252 (2004) 189 [arXiv:hep-th/0312171]. 
[9] F. Cachazo, P. Svrcek and E. Witten, JHEP 0409 (2004) 006 [arXiv:hep-th/0403047].

[10] S. J. Parke and T. R. Taylor, Phys. Rev. Lett. 56 (1986) 2459.

[11] R. Britto, F. Cachazo and B. Feng, Nucl. Phys. B 715 (2005) 499 [arXiv:hep-th/0412308].

[12] R. Britto, F. Cachazo, B. Feng and E. Witten, Phys. Rev. Lett. 94 (2005) 181602 [arXiv:hep-th/0501052].

[13] Z. Bern, L. J. Dixon and D. A. Kosower, arXiv:hep-ph/0507005.

[14] Z. Bern, L. J. Dixon and D. A. Kosower, Phys. Rev. D 71 (2005) 105013 [arXiv:hep-th/0501240].

[15] S. D. Badger, E. W. N. Glover and V. V. Khoze, arXiv:hep-th/0507161.

[16] S. D. Badger, E. W. N. Glover, V. V. Khoze and P. Svrcek, arXiv:hep-th/0504159

[17] D. Forde and D. A. Kosower, arXiv:hep-th/0507292

[18] K. Hagiwara and D. Zeppenfeld, Nucl. Phys. B 274 (1986) 1.

[19] H. Tanaka, Comput. Phys. Commun. 58 (1990) 153.

[20] A. Ballestrero, E. Maina and S. Moretti, Nucl. Phys. B 415 (1994) 265 [arXiv:hep-ph/9212246].

A. Ballestrero and E. Maina, Phys. Lett. B 350 (1995) 225 [arXiv:hep-ph/9403244].

[21] S. D. Badger, E. W. N. Glover and V. V. Khoze, JHEP 0503 (2005) 023 [arXiv:hep-th/0412275].

[22] L. J. Dixon, E. W. N. Glover and V. V. Khoze, JHEP 0412 (2004) 015 [arXiv:hep-th/0411092]. 